Survey on the Disaster Prevention System in Rural Areas and Isolated Islands: Preparing for the Nankai Trough Earthquake

Dr. Takamitsu Kodama, Mr. Kazuhiro Ozawa, Dr. Takashi Nakagawa

Aichi Medical University, Nagakute, Japan

Introduction: An expert government committee in Japan decided to revise the chance of a Nankai Trough earthquake in the next 30 years from $70 \%$ to between 70 and $80 \%$ in 2017. If a mega-earthquake occurs, medical institutions in disaster regions are required to perform self-contained activities during the super-acute phase. Human damage varies depending on whether medical functions can be sustained, particularly in rural areas and isolated islands. Here we examined actual situations.

Aim: To identify the issues of a disaster medical system in rural areas and isolated islands that need solving.

Methods: Regarding disaster preparedness planning, we conducted a survey on 10 hospitals undesignated as disaster key hospitals of remote area medical care bases (survey group), and 69 hospitals designated as disaster key hospitals (control group) in three prefectures in the Tokai region (Aichi, Mie, and Shizuoka).

Results: We received responses from four hospitals in the survey group and 52 hospitals in the control group. The hospitals in the survey group responded that they could accept 74 severe casualties and 85 moderate casualties. We identified problems such as insufficient stockpiling of fuel, water, and oxygen, and lack of a prioritized lifeline supply contract.

Discussion: It was predicted that human damage would be relatively minor given smaller populations in rural areas and isolated islands in the Tokai region. However, the number of patients would exceed their acceptance capacity. Moreover, the system for sustaining infrastructure is not adequate for providing medical services. Thus, it was indicated that these regions would be isolated in terms of disaster measures. It is imperative to establish a disaster medical system in rural areas and isolated islands that lack adequate disaster medical systems to manage Nankai Trough earthquakes.

Prehosp Disaster Med 2019;34(Suppl. 1):s170

doi:10.1017/S1049023X19003881

\section{Susceptibility of Host Immunity to Infectious Diseases in the Spacecraft Environment}

Miss Vienna Tran

School of Medicine, The University of Adelaide, Adelaide, Australia

Introduction: The principles of Disaster and Emergency Medicine are applicable beyond the confines of planet Earth. With the accelerating rate of climate change, natural disasters, and overpopulation, as well as the innate human appetite for knowledge and technological advancement, there has recently been an increased interest in the prospect of long-duration spaceflight with a view to colonize extra-terrestrial bodies, such as Mars. However, there is a need to understand the risk of adverse medical events in the hostile environment of space.
For example, previous incidences of infectious disease and immune dysregulation during a short-term mission have threatened to jeopardize the crew dynamic and the mission objectives. The risk of infectious diseases to the astronaut is one of the many knowledge gaps that must be addressed before long-duration flight is considered.

Aim: To review how spaceflight impacts an astronaut's in-flight susceptibility to infectious diseases.

Methods: Research was guided by the Microbiology section of the NASA Human Research Roadmap Program. Search terms in the University of Adelaide Library Search database collection included: "infectious diseases + spaceflight," "astronaut + immunity," "analog," and "inflammatory marker."

Results: Studies that have been conducted in-flight and on Earth demonstrate that both the astronaut and the microbe are affected by spaceflight. Stress, microgravity, and the isolated nature of the spacecraft have been found to compromise the immunity of the astronaut, as shown by reduced $T$ cell counts and increased viral shedding of dormant viruses. Microbes have demonstrated rapid adaptation mechanisms, including genetic mutation and increased virulence.

Discussion: This paper identifies a significant need for further research into host immunity during spaceflight to mitigate infectious disease risk. It is recommended that in-situ studies and terrestrial space analogs are most effective and that current knowledge on the principles of wilderness and expedition medicine be applied where possible.

Prehosp Disaster Med 2019;34(Suppl. 1):s170

doi:10.1017/S1049023X19003893

The System of Disaster Dispatch Team Corresponding to the Nuclear Disaster of Nagasaki University

Dr. Yoshibiro Nozaki

Nagasaki University Hospital, Nagasaki, Japan

Introduction: After accidents of Fukushima Daiichi power plant, the Japanese Government distinguished some medical institutions corresponding to the nuclear disaster by roles and functions. Nuclear Regulation Authority is managing these medical institutions. The Nagasaki University was designated as two centers for "the advanced radiation emergency medical support" and "nuclear emergency medical support". We established "Headquarters for Nuclear Disaster Response and Preparedness in Nagasaki University" (NDRP) and prepared for emergency ordinarily. The staff of headquarters are mainly concentrating their power on the network construction and joint training with each facility. We are improving the dispatch system of nuclear emergency medical assistance team, but some problems were found through the experiences of some trainings. Aim: To stimulate discussion and listen to opinions from several facilities.

Methods: The dispatch system of nuclear emergency medical assistance team imitated the system of Japan Disaster Medical Assistance Team (DMAT). Specifically, activity days of all teams are limited, and all teams should come under the command of the head of support acceptance medical institution of a disaster area. Particularly the main duties of the dispatch 
team, which is sent from the nuclear emergency medical support center, are unification and adjustment of the team activities from other facilities. Some other duties include offering appropriate medical care to patients at the disaster area and support of patients' transportation from the hospital at the disaster area to "advanced radiation emergency medical support center" or "nuclear emergency medical support center."

Discussion: In training the many facilities that participated, we realized that we couldn't proceed with each activity quickly and smoothly without support from the nuclear emergency medical assistance teams from outside the boundaries of disaster areas. We need to clarify the problems that are obtained from trainings and improve the current system corresponding to a nuclear disaster with efficiency.

Prehosp Disaster Med 2019;34(Suppl. 1):s170-s171

doi:10.1017/S1049023X1900390X

\section{A Systematic Review of Earthquake-Related Head Injuries} Dr. Yutaka Igarashi ${ }^{1}$, Ms Narumi Matsumoto ${ }^{2}$,

Dr. Tatsubiko Kubo ${ }^{3}$, Dr. Ryuta Nakae ${ }^{1}$, Dr. Shoji Yokobori ${ }^{1}$, Professor Hiroyuki Yokota ${ }^{1}$

1. Department of Emergency and Critical Care Medicine, Nippon Medical School Hospital, Tokyo, Japan

2. Faculty of Medicine, Nippon Medical School, Tokyo, Japan

3. Department of Environmental Epidemiology, Institute of Industrial Ecological Sciences, University of Occupational and Environmental Health, Kita-kyushu, Japan

Introduction: Earthquakes have killed around 800,000 people globally in the past 20 years, with head injury being the main cause of mortality and morbidity.

Aim: To conduct a systematic review to determine the characteristics of head injuries after earthquakes for better disaster preparedness and management.

Methods: All publications related to head injuries and earthquakes were searched using Pubmed, Web of Science, the Cochrane Library, and Ichushi.

Results: Thirty-six articles were included in the analysis. Head injury was the third most common cause of injury among survivors of earthquakes. The most common injury after an earthquake occurred was in the lower extremities (36.2\%), followed by the upper extremities (19.9\%), head (16.6\%), spine (13.3\%), chest (11.3\%), and abdomen (3.8\%). Earthquake-related head injuries were predominantly caused by a blunt strike (79\%), and were more frequently associated with soft tissue injury compared to non-earthquake-related head injuries and less frequently with intracranial hemorrhage. The mean age of patients with earthquake-related head injuries was 32.6 years, and $55.1 \%$ of sufferers were male. The most common earthquake-related head injury was laceration or contusion (59.2\%) while epidural hematoma was most common among inpatients with intracranial hemorrhage after an earthquake (9.5\%). Early wound irrigation and debridement and antibiotics administration are needed to decrease the risk of infection. Mortality due to earthquake-related head injuries was $5.6 \%$.
Discussion: Head injury was the main cause of mortality and morbidity after an earthquake. The characteristics of earthquake-related head injuries differed from those of non-earthquake-related head injuries, including the frequency of multiple injuries, and occurrence of contaminated soft tissue injury and epidural hematoma. This knowledge is important for determining demands for neurosurgery and for adequate management of patients, especially in resource-limited conditions.

Prehosp Disaster Med 2019;34(Suppl. 1):s171

doi:10.1017/S1049023X19003911

\section{Tabletop Simulation Exercise of Critically Ill Patient} Evacuation from a Hospital Fire

Ms Jiyoung Noh', Professor Hyun Soo Chung ${ }^{2}$, Ms Hye Mi Jin ${ }^{1}$, Ms Jayoung Hur ${ }^{1}$, Ms Min Ji Kim ${ }^{1}$, Ms Chan Mi Kang ${ }^{1}$,

Ms Ga Hyun Lee

1. Center for Disaster Relief, Training, and Research, Yonsei University Severance Hospital, Seoul, South Korea

2. Department of Emergency Medicine, Yonsei University College of Medicine, Seoul, South Korea

Introduction: Recent hospital fire incidents in South Korea have heightened the importance of patient evacuation. Moving patients from an intensive care unit (ICU) or emergency department (ED) setting is a challenge due to the complexity of moving acutely unwell patients who are reliant on invasive monitoring and organ support. Despite the importance of patient evacuation, the readiness of ICU and ED for urgent evacuation has not been assessed.

Aim: To enhance the readiness and competencies of workers from ICU and ED in the evacuation of patients during a simulated tabletop fire exercise.

Methods: A tabletop simulation exercise was developed by the Center for Disaster Relief, Training, and Research referencing the fire evacuation manual developed by the hospital's ICU and ED. The scenario consisted of evacuating patients horizontally and vertically from each department. The participants' actions were assessed using a checklist. A debriefing was completed after the exercise to discuss the gaps observed. A post-survey questionnaire was used to evaluate the exercise and assess the perception changes of the participants. All pre-to-post differences within subjects were analyzed with paired t-tests.

Results: A total of 22 and 29 people participated in the exercise from ICU and ED, respectively. Knowledge and confidence improved post-exercise for both ICU and ED scenarios $(p<0.05)$. Course satisfaction was 7.9 and 8.7, respectively for ICU and ED exercise. Correct performance rates for ICU and $\mathrm{ED}$ were $59 \%$ and 58\%, respectively. Common gaps noted for both ICU and ED were wearing protective masks, patient hand-over communication, and preparation for resources.

Discussion: There need to be exercises to recognize system gaps in place for hospital fire evacuation preparedness. Tabletop simulation exercises are ideal tools for this purpose. Although this was a short 90-minute exercise, this increased familiarity with the evacuation plan, tested the plan, and allowed for identification of gaps.

Prehosp Disaster Med 2019;34(Suppl. 1):s171

doi:10.1017/S1049023X19003923 